\title{
Long time behavior of a tumor-immune system competition model perturbed by environmental noise
}

Ying Li and Dongxi Li*

\section{"Correspondence:}

dxli0426@126.com

College of Mathematics, Taiyuan

University of Technology, 79 Yingze

West, Taiyuan, Shanxi 030024, China

\begin{abstract}
This paper investigates the long time behavior of tumor cells evolution in a tumor-immune system competition model perturbed by environmental noise. Sufficient conditions for extinction, stochastic persistence, and strong persistence in the mean of tumor cells are derived by constructing Lyapunov functions. The study results show that environmental noise can accelerate the extinction of tumor cells under immune surveillance of effector cells, which means that noise is favorable for the extinction of tumor in this condition. Finally, numerical simulations are introduced to support our results.
\end{abstract}

Keywords: tumor-immune system competition model; environmental noise; extinction; persistence

\section{Introduction}

Cancer is becoming the leading cause of death around the world, but our cognition of its causes, methods of prevention and cure are still in its infancy. One great method that has shown its potential in our better understanding of such a complicated biological problem is mathematical modeling [1]. Tumor immune models have existed since the early 1990s. Researchers have proposed various modeling approaches using ordinary and delayed differential equations [2-5]. A good summary of early works of tumor immune dynamics can be found in [6]. A detailed review of non-spatial models described by ODEs is published by Eftimie [7]. A simple but classical mathematical model of a cell mediated response to a growing tumor cell is proposed and analyzed by Kuznetsov and Taylor [8]; it takes into account the penetration of tumor cells by effector cells as well as the inactivation of effector cells. Their model can be applied to describe two different mechanisms of the tumor: tumor dormancy and sneaking through. Galach [9] firstly simplified the Kuznetsov-Taylor model by replacing the Michaelis-Menten form with a Lotka-Volterra form for the immune reactions. Then time delay was considered in the simplified model, and a state of the returning tumor was observed. More complete bibliography about the evolution of a cell and the relevant role of cellular phenomena in directing the body toward recovery or toward illness can be found in $[10,11]$. The detailed descriptions of virus, antivirus, and body dynamics are available in [12-15].

(c) The Author(s) 2017. This article is distributed under the terms of the Creative Commons Attribution 4.0 International License (http://creativecommons.org/licenses/by/4.0/), which permits unrestricted use, distribution, and reproduction in any medium, provided you give appropriate credit to the original author(s) and the source, provide a link to the Creative Commons license, and indicate if changes were made. 
Conventional treatments of cancer, such as radiotherapy and chemotherapy, usually act on the tumor cells themselves, and although these conventional treatments respond early, recurrences and drug resistance often occur late in the course of long-term treatment. Cancer immunotherapy has recently gained exciting progress. In contrast to conventional therapies, immunotherapy elicits immune system antitumor responses by acting on the immune system. Immune-Checkpoint Inhibitors clinical trials have shown a greater sustained response than conventional chemotherapy. The positive response of immunotherapy generally depends on the interaction of tumor cells and the immune regulation in the tumor microenvironment. In fact, tumor microenvironment is inevitably affected by environmental noise, which is an important component in realism. Nowadays, noise dynamics have been widely studied in different fields such as epidemic model [16], nervous system [17], genetic regulatory system [18-20], bistable system [21-23], chaotic system [24, 25], etc. In the last years, stochastic growth models for cancer cells were studied in [26-30], in which Lyapunov exponent method and Fokker-Planck equation method are used to investigate the stability of the stochastic model by numerical simulations. The goal of this paper is to explore the long time behavior of tumor and effector cells in the tumor-immune system competition model perturbed by environmental noise. One of many advantages of our paper is that we initially make use of the methods of Itô's formula and Lyapunov function to derive and analyze the properties of a stochastic tumor-immune system competition model. The other advantage of this paper is that the conditions for extinction, stochastic persistence, and strong persistence in the mean of tumor cells are established by strict mathematical proofs. Accordingly, the sufficient conditions for extinction and persistence could provide us more effective and precise therapeutic schedule to eliminate tumor cells and improve the treatment of cancer.

This paper is organized as follows. In Section 2, we introduce a mathematical model. In Section 3, we establish the sufficient conditions for extinction, stochastic persistence, and strong persistence in the mean of tumor cells. Numerical simulations are presented in Section 4, and they are used to verify and illustrate the theorems of Section 3. In Section 5, we discuss the conclusions and future directions of the research.

\section{Mathematical model}

When unknown tissues, organisms, or tumor cells appear in a body, the immune system tries to identify them and, if it succeeds, it attempts to eliminate them. The immune system response consists of two distinct interacting responses: the cellular response and the humoral response. The cellular response is carried by $\mathrm{T}$ lymphocytes. The humoral response is mediated by B lymphocytes. The dynamics of the antitumor immune response in vivo is complicated and not well understood.

Once the tumor cells are identified, the immune response begins. Then tumor cells are caught by macrophages, which can be found in all tissues in the body and circulate in the blood stream. Macrophages absorb tumor cells, eat them, and release a series of cytokines which activate $\mathrm{T}$ helper cells (i.e., a subpopulation of $\mathrm{T}$ lymphocytes). Activated $\mathrm{T}$ helper cells coordinate the counterattack. Thelper cells can also be directly stimulated to interact with antigens. These helper cells cannot kill tumor cells, but they send urgent biochemical signals to a special type of T lymphocytes called natural killers (NKs). T cells begin to multiply and release other cytokines that further stimulate more T cells, B cells, and NK cells. As the number of $\mathrm{B}$ cells increases, $\mathrm{T}$ helper cells send a signal to start the production 
Figure 1 Kinetic scheme describing interactions between ECs and TCs (see [9]).

$$
E+T \underset{k_{-1}}{\stackrel{k_{1}}{\rightleftarrows} C \stackrel{k_{2}}{\rightleftarrows} E+T^{*}} \begin{aligned}
& E^{*}+T
\end{aligned}
$$

of antibodies. Antibodies circulate in the blood and are attached to tumor cells, which implies that the tumor cells are more quickly engulfed by macrophages or killed by NK cells. Like all T cells, NK cells are programmed to identify one specific type of infected cell or cancer cell. NK cells are lethal and constitute a vital line of the defense.

The model presented in [8] describes the response of effector cells (ECs) to the growth of tumor cells (TCs). This model differs from others because it takes into account the penetration of TCs by ECs, which simultaneously causes the inactivation of ECs. It is assumed that interactions between ECs and TCs in vitro can be described by the kinetic scheme shown in Figure 1, where $E, T, C, E^{*}$, and $T^{*}$ are the local concentrations of ECs, TCs, ECTC complexes, inactivated ECs, and lethally hit TCs, respectively. $k_{1}$ and $k_{-1}$ denote the rates of bindings of ECs to TCs and the detachment of ECs from TCs without damaging TCs, $k_{2}$ is the rate at which EC-TC interactions program TCs for lysis, and $k_{3}$ is the rate at which EC-TC interactions inactivate ECs.

Kuznetsov and Taylor's model [8] is as follows:

$$
\left\{\begin{array}{l}
\frac{d E}{d t}=s+F(C, T)-d_{1} E-k_{1} E T+\left(k_{-1}+k_{2}\right) C, \\
\frac{d T}{d t}=a T(1-b T)-k_{1} E T+\left(k_{-1}+k_{3}\right) C, \\
\frac{d C}{d t}=k_{1} E T-\left(k_{-1}+k_{2}+k_{3}\right) C, \\
\frac{d E^{*}}{d t}=k_{3} C-d_{2} E^{*} \\
\frac{d T^{*}}{d t}=k_{2} C-d_{3} T^{*}
\end{array}\right.
$$

where $s$ is the normal (i.e., not increased by the presence of the tumor) rate of the flow of adult ECs into the tumor site. $F(C, T)$ describes the accumulation of ECs in the tumor site; $d_{1}, d_{2}$, and $d_{3}$ are the coefficients of the processes of destruction and migration for $E, E^{*}$, and $T^{*}$, respectively; $a$ is the coefficient of the maximal growth of tumor; and $1 / b$ is the environment capacity.

It is claimed in [8] that experimental observations motivate the approximation $\frac{d C}{d t} \approx 0$. Therefore, it is assumed that $C \approx K E T$, where $K=\frac{k_{1}}{k_{2}+k_{3}+k_{-1}}$, and the model can be reduced to two equations which describe the behavior of ECs and TCs only. Moreover, in [9] it is suggested that the function $F$ should be in the form $F(C, T)=F(E, T)=\theta E T$. Therefore, model (1) takes the form

$$
\left\{\begin{array}{l}
\frac{d E}{d t}=s+\alpha_{1} E T-d E \\
\frac{d T}{d t}=a T(1-b T)-n E T
\end{array}\right.
$$

where $\alpha_{1}=\theta-m$, and $a, b, s$ have the same meanings as those in (1); $n=K k_{2}, m=K k_{3}$, and $d=d_{1}$, respectively. All coefficients except $\alpha_{1}$ are positive. $\alpha_{1}$ is the immune response to the appearance of the tumor cells, its sign depends on the relation between $\theta$ and $m$. If the stimulation coefficient of the immune system exceeds the neutralization coefficient of ECs during the formation of EC-TC complexes, then $\alpha_{1}>0$. We use the dimensionless 
form of model (2),

$$
\left\{\begin{array}{l}
\frac{d x}{d t}=\varepsilon+\omega x y-\delta x \\
\frac{d y}{d t}=\alpha y(1-\beta y)-x y
\end{array}\right.
$$

where $x$ denotes the dimensionless density of ECs; $y$ stands for the dimensionless density of the population of TCs; $E_{0}$ and $T_{0}$ are the initial value. $\alpha=\frac{a}{n T_{0}}, \beta=b T_{0}, \delta=\frac{d}{n T_{0}}, \varepsilon=$ $\frac{s}{n E_{0} T_{0}}$, and $\omega=\frac{\alpha_{1}}{n}$ represent the immune response to the appearance of the tumor cells (i.e., immune coefficient). In this paper we consider the case of $\omega>0$, which means that immune response is positive.

In [9], system (3) always has the equilibrium $P_{0}=\left(\frac{\varepsilon}{\delta}, 0\right)$. If $\omega>0$ and $\alpha \delta<\varepsilon$, then $P_{0}$ is the unique equilibrium of (3) and it is globally stable. If $\omega>0$ and $\alpha \delta>\varepsilon$, then $P_{0}$ is unstable, and there is an equilibrium

$$
P^{*}=\left(\frac{-\alpha(\beta \delta-\omega)+\sqrt{\Delta}}{2 \omega}, \frac{\alpha(\beta \delta+\omega)-\sqrt{\Delta}}{2 \alpha \beta \omega}\right)
$$

of (3), which is globally stable, and $\Delta=\alpha^{2}(\beta \delta-\omega)^{2}+4 \alpha \beta \varepsilon \omega$.

In fact, tumor microenvironment is complex, and the growth of tumor cells is influenced by many environmental factors [31], e.g., the supply of oxygen and nutrients, the degree of vascularization of tissues, the immunological state of the host, chemical agents, temperature, etc. A tiny variation of parameters will change the evolutionary process of tumor cells. So, it is inevitable to consider the tumor-immune system competition model with environmental noise. In this paper, taking into account the effect of randomly fluctuating environment, we assume that fluctuations in the environment will mainly affect the immune coefficient $\omega$

$$
\omega \rightarrow \omega+\sigma \dot{B}(t)
$$

where $B(t)$ is standard Brownian motions with $B(0)=0$, and with the intensity of white noise $\sigma^{2}>0$. The stochastic version corresponding to the deterministic model (3) takes the following form:

$$
\left\{\begin{array}{l}
d x(t)=(\varepsilon+\omega x(t) y(t)-\delta x(t)) d t+\sigma x(t) y(t) d B(t), \\
d y(t)=\left(\alpha y(t)-\alpha \beta y^{2}(t)-x(t) y(t)\right) d t
\end{array}\right.
$$

where all the parameters are positive and bounded. For convenience of expression, we define the following notions:

$$
\langle f(t)\rangle=\frac{1}{t} \int_{0}^{t} f(s) d s ; \quad f^{*}=\limsup _{t \rightarrow+\infty} f(t) ; \quad f_{*}=\liminf _{t \rightarrow+\infty} f(t) ; \quad t \wedge \tau_{k}=\min \left(t, \tau_{k}\right) .
$$

\section{Long time behavior of the stochastic model}

Our primary interests in tumor dynamics are the extinction and survival of tumors induced by environmental fluctuations. In order to study the extinction and survival, we need some appropriate definitions about extinction and persistence. Here we adopt the 
concepts of extinction, stochastically permanent [32], and strong persistence in the mean [33]. In addition, some of our proofs are motivated by the works Liu [33], Mao [34], and Zhao [16]. The useful definitions are as follows:

(1) The tumor cells $y(t)$ will go to extinction a.s. if $\lim _{t \rightarrow+\infty} y(t)=0$.

(2) The tumor cells $y(t)$ will be stochastically permanent a.s. if there are constants $N>0$ and $M>0$ such that $\mathcal{P}_{*}\{y(t) \geq N\} \geq 1-\xi$ and $\mathcal{P}_{*}\{y(t) \leq M\} \geq 1-\xi$.

(3) The tumor cells $y(t)$ will be strong persistent in the mean a.s. if $\langle y(t)\rangle_{*}>0$.

Next we establish the sufficient conditions of extinction and persistence for our model.

Lemma 1 For any positive initial value $\left(x_{0}, y_{0}\right)$, if $0<x_{0}<\frac{1}{\beta}$, the solution of (4) obeys

$$
x(t)<\frac{1}{\beta}, \quad y(t) \leq \max \left\{y_{0}, \frac{1}{\beta}\right\} \quad \text { a.s. }
$$

Proof According to the second equation of model (4), we have

$$
y=\frac{\alpha-x}{\alpha \beta+\left(\frac{\alpha-x}{y_{0}}-\alpha \beta\right) e^{-(\alpha-x) t}} .
$$

Firstly, we discuss $y$ based on the different value range of $x$.

(i) When $0<\alpha-x$ and $\alpha \beta y_{0} \leq \alpha-x$ (i.e., $0<x \leq \alpha\left(1-\beta y_{0}\right)$ ),

$$
\begin{aligned}
y & =\frac{1}{\frac{\alpha \beta}{\alpha-x}+\left(\frac{1}{y_{0}}-\frac{\alpha \beta}{\alpha-x}\right) e^{-(\alpha-x) t}} \\
& =\frac{1}{\frac{\alpha \beta}{\alpha-x}+\frac{\alpha \beta}{\alpha-x}\left(\frac{\alpha-x}{\alpha \beta y_{0}}-1\right) e^{-(\alpha-x) t}} \\
& \leq \frac{1}{\frac{\alpha \beta}{\alpha-x}} \\
& \leq \frac{1}{\beta} .
\end{aligned}
$$

(ii) When $0<\alpha-x<\alpha \beta y_{0}$ (i.e., $\alpha\left(1-\beta y_{0}\right)<x<\alpha$ ),

$$
\begin{aligned}
y & =\frac{\alpha-x}{\alpha \beta+\left(\frac{\alpha-x}{y_{0}}-\alpha \beta\right) e^{-(\alpha-x) t}} \\
& <\frac{\alpha-x}{\alpha \beta+\left(\frac{\alpha-x}{y_{0}}-\alpha \beta\right)}=y_{0} .
\end{aligned}
$$

(iii) When $\alpha-x \leq 0$ (i.e., $x \geq \alpha$ ),

$$
\begin{aligned}
y & =\frac{x-\alpha}{-\alpha \beta+\left(\alpha \beta-\frac{\alpha-x}{y_{0}}\right) e^{-(\alpha-x) t}} \\
& =\frac{x-\alpha}{-\alpha \beta+\left(\alpha \beta+\frac{x-\alpha}{y_{0}}\right) e^{-(\alpha-x) t}} \\
& \leq \frac{x-\alpha}{-\alpha \beta+\alpha \beta+\frac{x-\alpha}{y_{0}}}=y_{0} .
\end{aligned}
$$


Consequently, we have proved $y(t) \leq \max \left\{y_{0}, \frac{1}{\beta}\right\}$. Then we will show that $x(t)$ is bounded. Applying Itô's formula [35] to the first equation of model (4), we get

$$
d \ln \left|\frac{1}{x-\frac{1}{\beta}}\right|=\frac{\beta(1-\beta x)(\varepsilon+\omega x y-\delta x)+0.5 \beta^{2} \sigma^{2} x^{2} y^{2}}{(\beta x-1)^{2}} d t-\frac{\beta \sigma x y}{\beta x-1} d B(t) .
$$

Integrating both sides from 0 to $t$, we can obtain

$$
x(t)-\frac{1}{\beta}=\frac{x_{0}-\frac{1}{\beta}}{e^{\int_{0}^{t} M_{1}(s) d s+\int_{0}^{t} M_{2}(s) d B(s)}},
$$

where $M_{1}(s)=\frac{\beta(1-\beta x(s))(\varepsilon+\omega x(s) y(s)-\delta x(s))+0.5 \beta^{2} \sigma^{2} x^{2}(s) y^{2}(s)}{(\beta x(s)-1)^{2}}$, and $M_{2}(s)=-\frac{\beta \sigma x(s) y(s)}{\beta x(s)-1}$.

Therefore,

$$
x(t)=\frac{x_{0}-\frac{1}{\beta}}{e^{\int_{0}^{t} M_{1}(s) d s+\int_{0}^{t} M_{2}(s) d B(s)}}+\frac{1}{\beta} .
$$

That is to say, if $x_{0}<\frac{1}{\beta}$, then $x(t)<\frac{1}{\beta}$ for all $t>0$.

Lemma 2 Let $f \in C[[0, \infty) \times \Omega,(0, \infty)]$ and $F(t) \in C([0, \infty) \times \Omega$, $R)$. If there exist positive constants $\lambda_{0}, \lambda$, and $T$ such that

$$
\ln f(t) \geq \lambda t-\lambda_{0} \int_{0}^{t} f(s) d s-F(t) \quad \text { a.s. }
$$

for all $t \geq T$, and $\lim _{t \rightarrow \infty} \frac{F(t)}{t}=0$ a.s., then

$$
\liminf _{t \rightarrow \infty} \frac{1}{t} \int_{0}^{t} f(s) d s \geq \frac{\lambda}{\lambda_{0}} \quad \text { a.s. }
$$

Proof The proof is similar to the proof of Lemma in [16]. Note that $\lim _{t \rightarrow \infty} \frac{F(t)}{t}=0$ a.s.; then, for arbitrary $k>0$, there exist $T_{0}=T_{0}(\omega)>0$ and a set $\Omega_{k}$ such that $P\left(\Omega_{k}\right) \geq 1-k$ and $\frac{F(t)}{t} \leq k$ for all $t \geq T_{0}, \omega \in \Omega_{k}$. Let $\bar{T}=\max \left\{T, T_{0}\right\}$ and

$$
\varphi(t)=\int_{0}^{t} f(s) d s \quad \text { for } t \geq \bar{T}, \omega \in \Omega_{k} .
$$

Since $f \in C[[0, \infty) \times \Omega,(0, \infty)]$, then $\varphi(t)$ is differentiable on $[\bar{T}, \infty)$ a.s. and

$$
\frac{d \varphi(t)}{d t}=f(t)>0 \quad \text { for } t \geq \bar{T}, \omega \in \Omega_{k} .
$$

Substituting $\frac{d \varphi(t)}{d t}$ and $\varphi(t)$ into (7), we obtain

$$
\ln \left(\frac{d \varphi(t)}{d t}\right) \geq \lambda t-\lambda_{0} \varphi(t)-F(t) \geq(\lambda-k) t-\lambda_{0} \varphi(t) \quad \text { for } t \geq \bar{T}, \omega \in \Omega_{k} \text {. }
$$

Thus

$$
e^{\lambda_{0} \varphi(t)} \frac{d \varphi(t)}{d t} \geq e^{(\lambda-k) t} \quad \text { for } t \geq \bar{T}, \omega \in \Omega_{k} .
$$


Integrating this inequality from $\bar{T}$ to $t$ results in

$$
\lambda_{0}^{-1}\left[e^{\lambda_{0} \varphi(t)}-e^{\lambda_{0} \varphi(\bar{T})}\right] \geq(\lambda-k)^{-1}\left[e^{(\lambda-k) t}-e^{(\lambda-k) \bar{T}}\right] .
$$

This inequality can be rewritten as

$$
e^{\lambda_{0} \varphi(t)} \geq \lambda_{0}(\lambda-k)^{-1}\left[e^{(\lambda-k) t}-e^{(\lambda-k) \bar{T}}\right]+e^{\lambda_{0} \varphi(\bar{T})} .
$$

Taking the logarithm on both sides yields

$$
\varphi(t) \geq \lambda_{0}^{-1} \ln \left[\lambda_{0}(\lambda-k)^{-1} e^{\lambda-k} t+\lambda_{\bar{T}}\right]
$$

where

$$
\lambda_{\bar{T}}=e^{\lambda_{0} \varphi(\bar{T})}-\lambda_{0}(\lambda-k)^{-1} e^{\lambda-k} \bar{T},
$$

or

$$
\int_{0}^{t} f(s) d s \geq \lambda_{0}^{-1} \ln \left[\lambda_{0}(\lambda-k)^{-1} e^{(\lambda-k) t}+\lambda_{\bar{T}}\right] \quad \text { for } t \geq \bar{T}, \omega \in \Omega_{k} .
$$

Dividing both sides by $t(\geq \bar{T}>0)$ gives

$$
\frac{1}{t} \int_{0}^{t} f(s) d s \geq \lambda_{0}^{-1} t^{-1} \ln \left[\lambda_{0}(\lambda-k)^{-1} e^{(\lambda-k) t}+\lambda_{\bar{T}}\right] .
$$

Taking the limit inferior on both sides and applying L'Hospital's rule on the right-hand side of this inequality, we obtain

$$
\liminf _{t \rightarrow \infty} \frac{1}{t} \int_{0}^{t} f(s) d s \geq \frac{\lambda-k}{\lambda_{0}} \quad \text { for } \omega \in \Omega_{k} .
$$

Letting $k \rightarrow 0$ yields

$$
\liminf _{t \rightarrow \infty} \frac{1}{t} \int_{0}^{t} f(s) d s \geq \frac{\lambda}{\lambda_{0}}, \quad \text { a.s. }
$$

Theorem 1 For any positive initial value $\left(x_{0}, y_{0}\right)$, particularly, $x_{0}<\frac{1}{\beta}$, Equation (4) has a positive unique global solution $(x(t), y(t))$ on $t \geq 0$ a.s.

Proof To get a unique global solution for any given initial value, the coefficients of the equation are generally required to satisfy the linear growth condition and the local Lipschitz condition [36]. However, the coefficients of model (4) do not satisfy the linear growth condition, so the solution may explode in a finite time. Since the coefficients of Equation (4) are locally Lipschitz continuous for any given initial value $\left(x_{0}, y_{0}\right) \in R_{+}^{2}$, there is a unique maximal local solution $(x(t), y(t))$ on $t \in\left[0, \tau_{e}\right]$, where $\tau_{e}$ is the explosion time [36]. To show this solution is global, we only need to show that $\tau_{e}=\infty$. To this end, let $k_{0}>0$ be sufficiently large so that $x_{0}, y_{0}$ all lie within the interval $\left[\frac{1}{k_{0}}, k_{0}\right]$. For each integer $k \geq k_{0}$, we define the stopping times $\tau_{k}=\inf \left\{t \in\left[0, \tau_{e}\right]: \min \{x(t), y(t)\} \leq \frac{1}{k}\right.$ or $\left.\max \{x(t), y(t)\} \geq k\right\}$. 
Clearly, $\tau_{k}$ is increasing as $k \rightarrow \infty$. Set $\tau_{\infty}=\lim _{k \rightarrow+\infty} \tau_{k}$, thus $\tau_{\infty} \leq \tau_{e}$ a.s. In other words, we only need to prove $\tau_{\infty}=\infty$. If this statement is false, there exist constants $T>0$ and $\varepsilon \in(0,1)$ so that $\mathcal{P}\left\{\tau_{\infty}<\infty\right\}>\varepsilon$. Thus there is an integer $k_{1}>k_{0}$ such that

$$
\mathcal{P}\left\{\tau_{k}<T\right\}>\varepsilon, \quad k>k_{1} .
$$

Define a $C^{2}$-function $V: R_{+}^{2} \rightarrow R_{+}$by $V(x, y)=(x-1-\ln x)+(y-1-\ln y)$. The nonnegativity of this function can be seen from $u-1-\ln u \geq 0, \forall u>0$. Let $k \geq k_{0}$ and $T>0$ be arbitrary. Applying Itô’s formula, we have

$$
\begin{aligned}
d V(x, y)= & \left(1-\frac{1}{x}\right) d x+\frac{1}{2 x^{2}}(d x)^{2}+\left(1-\frac{1}{y}\right) d y+\frac{1}{2 y^{2}}(d y)^{2} \\
= & {\left[\varepsilon+(\omega-1) x y+(1-\delta) x-\varepsilon x^{-1}+(\alpha \beta-\omega+\alpha) y+\delta\right.} \\
& \left.\left.+\left(0.5 \sigma^{2}-\alpha \beta\right) y^{2}-\alpha\right)\right] d t+\sigma\left(1-x^{-1}\right) x y d B(t) \\
\leq & L d t+\frac{\sigma}{\beta}(x-1) d B(t) .
\end{aligned}
$$

Here, $L$ is a positive constant, and in the proof of the last inequality, we have used Lemma 1 (i.e., for $\forall t \geq 0, x(t)$ and $y(t)$ are bounded). The inequality in (9) implies that

$$
\int_{0}^{\tau_{k} \wedge T} d V(x(t), y(t)) \leq \int_{0}^{\tau_{k} \wedge T} L d t+\int_{0}^{\tau_{k} \wedge T} \frac{\sigma}{\beta}(x(s)-1) d B(s)
$$

Taking expectation on both sides of the above inequality, we can obtain

$$
E V\left(x\left(\tau_{k} \wedge T\right), y\left(\tau_{k} \wedge T\right)\right) \leq V\left(x_{0}, y_{0}\right)+L E\left(\tau_{k} \wedge T\right) \leq V\left(x_{0}, y_{0}\right)+L T
$$

Let $\Omega_{k}=\left\{\tau_{k} \wedge T\right\}$, then by Inequality (8) we have $P\left(\Omega_{k}\right) \geq \varepsilon$. Note that for any $\omega \in \Omega_{k}$, $x\left(\tau_{k}, \omega\right), y\left(\tau_{k}, \omega\right)$ equals either $k$ or $1 / k$, hence $V\left(x\left(\tau_{k}, \omega\right), y\left(\tau_{k}, \omega\right)\right)$ is no less than $\min \{2(k-$ $\left.1-\ln k), 2\left(\frac{1}{k}-1+\ln k\right), k+\frac{1}{k}-2\right\}$. By Formula (10) we have

$$
\begin{aligned}
V\left(x_{0}, y_{0}\right)+L T & \geq E\left[1_{\Omega_{k}} V\left(x\left(\tau_{k}\right), y\left(\tau_{k}\right)\right)\right] \\
& \geq \varepsilon \min \left\{2(k-1-\ln k), 2\left(\frac{1}{k}-1+\ln k\right), k+\frac{1}{k}-2\right\},
\end{aligned}
$$

where $1_{\Omega_{k}}$ is the indicator function of $\Omega_{k}$. Letting $k \rightarrow \infty$, the contradiction $\infty>$ $V\left(x_{0}, y_{0}\right)+L T=\infty$ exists, which completes the proof.

Remark 1 In order to guarantee the existence and uniqueness of the solution of model (4), we discuss the extinction and persistence of $y(t)$ under the condition $x_{0}<\frac{1}{\beta}$ below.

Theorem 2 Let $(x(t), y(t))$ be the solution of system (4) with positive initial value $\left(x_{0}, y_{0}\right)$. If $\alpha \delta<\varepsilon$, then

$$
\lim _{t \rightarrow+\infty} x(t)=\frac{\varepsilon}{\delta}, \quad \lim _{t \rightarrow+\infty} y(t)=0 .
$$


Proof An integration of the first equation of model (4) yields

$$
\frac{x(t)-x_{0}}{t}=\varepsilon+\omega\langle x(t) y(t)\rangle-\delta\langle x(t)\rangle+\frac{\sigma}{t} \int_{0}^{t} x(s) y(s) d B(s) .
$$

We compute that

$$
\begin{aligned}
\langle x(t)\rangle & =\frac{\varepsilon}{\delta}+\frac{\omega}{\delta}\langle x(t) y(t)\rangle+\frac{\sigma}{\delta t} \int_{0}^{t} x(s) y(s) d B(s)-\frac{x(t)-x_{0}}{\delta t} \\
& \geq \frac{\varepsilon}{\delta}+\frac{N_{1}(t)}{t}-\frac{x(t)-x_{0}}{\delta t}
\end{aligned}
$$

where $N_{1}(t)=\frac{\sigma}{\delta} \int_{0}^{t} x(s) y(s) d B(s)$, which is a local continuous martingale and $N_{1}(0)=0$. Moreover,

$$
\limsup _{t \rightarrow \infty} \frac{\left\langle N_{1}, N_{1}\right\rangle_{t}}{t}<\frac{\sigma^{2}}{\delta^{2} \beta^{4}}<\infty \text { a.s. }
$$

By the strong law of large numbers for local martingales [36], we obtain

$$
\lim _{t \rightarrow \infty} \frac{N_{1}(t)}{t}=0 \quad \text { a.s. }
$$

Taking the limit inferior on both sides of (12), we have

$$
\lim _{t \rightarrow+\infty}\langle x(t)\rangle_{*} \geq \frac{\varepsilon}{\delta} \quad \text { a.s. }
$$

Applying Itô's formula to the second equation of model (4), we get

$$
d \ln y=(\alpha-\alpha \beta y-x) d t
$$

Integrating this from 0 to $t$ and dividing by $t$ on both sides, we have

$$
\begin{aligned}
\frac{\ln y(t)-\ln y_{0}}{t} & =\alpha-\alpha \beta\langle y(t)\rangle-\langle x(t)\rangle \\
& \leq \alpha-\langle x(t)\rangle .
\end{aligned}
$$

Taking the limit superior on both sides of (15) and substituting (14) into (15) yields

$$
\limsup _{t \rightarrow \infty} \frac{\ln y(t)}{t} \leq \alpha-\frac{\varepsilon}{\delta} .
$$

If the condition $\alpha \delta<\varepsilon$ is satisfied, then

$$
\limsup _{t \rightarrow \infty} \frac{\ln y(t)}{t}<0 \quad \text { a.s. }
$$

which implies

$$
\lim _{t \rightarrow \infty} y(t)=0 \quad \text { a.s. }
$$


Applying Itô's formula to the first equation of model (4) leads to

$$
d \ln \frac{1}{x}=\left(-\varepsilon \frac{1}{x}-\omega y+\delta+0.5 \sigma^{2} y^{2}\right) d t-\sigma y d B(t)
$$

Integrating this from 0 to $t$, we have

$$
\ln \frac{1}{x(t)}-\ln \frac{1}{x_{0}}=\delta t+\int_{0}^{t}\left[-\varepsilon \frac{1}{x(s)}-\omega y(s)+0.5 \sigma^{2} y^{2}(s)\right] d s-\sigma \int_{0}^{t} y(s) d B(s) .
$$

Set $N_{2}(t)=\int_{0}^{t} \sigma y(s) d B(s)$, whose quadratic variation is

$$
\left\langle N_{2}(t), N_{2}(t)\right\rangle=\int_{0}^{t} \sigma^{2} y^{2}(s) d s
$$

By virtue of the exponential martingale inequality [36], for any positive constants $T$, $a$ and $b$, we have

$$
\mathcal{P}\left\{\sup _{0 \leq t \leq T}\left[N_{2}(t)-\frac{a}{2}\left\langle N_{2}(t), N_{2}(t)\right\rangle\right]>b\right\} \leq e^{-a b} .
$$

Choose $T=n, a=1, b=2 \ln n$, we get

$$
\mathcal{P}\left\{\sup _{0 \leq t \leq n}\left[N_{2}(t)-\frac{1}{2}\left\langle N_{2}(t), N_{2}(t)\right\rangle\right]>2 \ln n\right\} \leq 1 / n^{2}
$$

An application of the Borel-Cantelli lemma [36] yields that for almost all $\omega \in \Omega$, there is a random integer $n_{0}=n_{0}(\omega)$ such that for $n \geq n_{0}$,

$$
\sup _{0 \leq t \leq n}\left[N_{2}(t)-\frac{1}{2}\left\langle N_{2}(t), N_{2}(t)\right\rangle\right] \leq 2 \ln n
$$

That is to say,

$$
N_{2}(t) \leq 2 \ln n+\frac{1}{2}\left\langle N_{2}(t), N_{2}(t)\right\rangle=2 \ln n+0.5 \int_{0}^{t} \sigma^{2} y^{2}(s) d s
$$

for all $0 \leq t \leq n, n \geq n_{0}$ a.s. Substituting the above inequality into (17) leads to

$$
\ln \frac{1}{x(t)} \geq \delta t-\varepsilon \int_{0}^{t} \frac{1}{x(s)} d s-\left(\omega \int_{0}^{t} y(s) d s+2 \ln n-\ln \frac{1}{x_{0}}\right)
$$

By use of Lemma 2, we have

$$
\langle 1 / x(t)\rangle_{*} \geq \frac{\delta}{\varepsilon}
$$

Namely,

$$
\langle x(t)\rangle^{*} \leq \frac{\varepsilon}{\delta} .
$$


Together (18) with (14), we see that

$$
\lim _{t \rightarrow \infty}\langle x(t)\rangle=\frac{\varepsilon}{\delta} .
$$

Applying L'Hospital's rule to the left side of (19), we can derive that

$$
\lim _{t \rightarrow \infty} x(t)=\frac{\varepsilon}{\delta}
$$

Theorem 3 If $\alpha \delta-\frac{\omega \alpha}{4 \beta}>\varepsilon$, then the tumor cells $y(t)$ will be stochastically permanent almost surely.

Proof We first show that for arbitrary fixed $\xi>0$, there exists a constant $M>0$ such that $\mathcal{P}_{*}(y(t) \leq M) \geq 1-\xi$. Define $V(y)=y^{q}$ for $y \in R_{+}$, where $0<q<1$. Then it follows from Itô's formula that

$$
\begin{aligned}
d V(y) & =q y^{q-1} d y+\frac{q(q-1)}{2} y^{q-2}(d y)^{2} \\
& =\left(q \alpha y^{q}-q \alpha \beta y^{q+1}-q x y^{q}\right) d t .
\end{aligned}
$$

Let $n_{0}$ be so large that $y_{0} \in\left[1 / n_{0}, n_{0}\right]$. For each integer $n \geq n_{0}$, define the stopping time $\tau_{n}=\inf \{t \geq 0: y(t) \notin(1 / n, n)\}$. Clearly, $\tau_{n} \rightarrow \infty$ almost surely as $n \rightarrow \infty$. Applying Itô's formula again to $\exp (t) V(y)$ gives

$$
\begin{aligned}
d(\exp (t) V(y)) & =\exp (t) V(y) d t+\exp (t) d V(y) \\
& =\exp (t) y^{q} d t+\exp (t)\left(q \alpha y^{q}-q \alpha \beta y^{q+1}-q x y^{q}\right) d t \\
& =\exp (t)\left(-q \alpha \beta y^{q+1}+(q \alpha+1) y^{q}-q x y^{q}\right) d t \\
& \leq \exp (t) K
\end{aligned}
$$

where $K$ is a positive constant. Integrating this inequality and then taking expectations on both sides, one can see that

$$
E\left[\exp \left(t \wedge \tau_{n}\right) y^{q}\left(t \wedge \tau_{n}\right)\right]-y_{0}^{q} \leq E \int_{0}^{t \wedge \tau_{n}} \exp (s) K d s
$$

Letting $n \rightarrow \infty$ yields $\exp (t) E\left[y^{q}(t)\right] \leq y_{0}^{q}+K(\exp (t)-1)$, or

$$
E\left[y^{q}(t)\right] \leq \exp (-t) y_{0}^{q}+K
$$

In other words, we have already shown that $\limsup _{t \rightarrow+\infty} E\left[y^{q}(t)\right] \leq K$. Thus, for any given $\xi>0$, let $M=K^{1 / q} / \xi^{1 / q}$, by virtue of Chebyshev's inequality, we can derive that

$$
\mathcal{P}\{y(t)>M\}=\mathcal{P}\left\{y^{q 2}(t)>M^{q 2}\right\} \leq \frac{E\left[y^{q}(t)\right]}{M^{q}},
$$

that is to say, $\mathcal{P}^{*}\{y(t)>M\} \leq \xi$. Consequently, $\mathcal{P}_{*}\{y(t) \leq M\} \geq 1-\xi$. 
Next we demonstrate that for arbitrary given $\xi>0$, there exists a constant $N>0$ such that $\mathcal{P}_{*}(y(t) \geq N) \geq 1-\xi$. Define $V_{1}(y)=y^{-2}$ for $y \in R_{+}$. Applying Itô's formula to $V_{1}(y)$ leads to

$$
\begin{aligned}
d V_{1}(y(t)) & =-2 y^{-3} d y+3 y^{-4}(d y)^{2} \\
& =-2 y^{-3}\left(\alpha y-\alpha \beta y^{2}-x y\right) d t \\
& =2 V_{1}(y)(-\alpha+\alpha \beta y+x) d t .
\end{aligned}
$$

Define $V_{2}(y)=\left(1+V_{1}(y)\right)^{\theta}$, where $\theta \in(1 / 4,1 / 2)$. Applying Itô's formula again to $V_{2}(y)$ gives

$$
\begin{aligned}
d V_{2}(y(t)) & =\theta\left(1+V_{1}(y(t))\right)^{\theta-1} d V_{1}+0.5 \theta(\theta-1)\left[1+V_{1}(y(t))\right]^{\theta-2}\left(d V_{1}\right)^{2} \\
& =\theta\left(1+V_{1}(y(t))\right)^{\theta-1}\left[2 V_{1}(y)(-\alpha+\alpha \beta y+x)\right] d t \\
& =\theta\left(1+V_{1}(y(t))\right)^{\theta-1}\left[-2(\alpha-x) V_{1}(y)+2 \alpha \beta V_{1}^{0.5}(y)\right] d t .
\end{aligned}
$$

An integration of system (4) is

$$
\left\{\begin{array}{l}
\frac{x(t)-x(0)}{t}=\varepsilon+\omega\langle x(t) y(t)\rangle-\delta\langle x(t)\rangle+\frac{\sigma}{t} \int_{0}^{t} x(s) y(s) d B(s), \\
\frac{y(t)-y(0)}{t}=\alpha\langle y(t)\rangle-\alpha \beta\left\langle y^{2}(t)\right\rangle-\langle x(t) y(t)\rangle .
\end{array}\right.
$$

According to (22), we have

$$
\begin{aligned}
\frac{x(t)-x(0)}{t}+\omega \frac{y(t)-y(0)}{t}= & \varepsilon-\delta\langle x(t)\rangle+\frac{\sigma}{t} \int_{0}^{t} x(s) y(s) d B(s)+\omega \alpha\langle y(t)\rangle \\
& -\omega \alpha \beta\left\langle y^{2}(t)\right\rangle .
\end{aligned}
$$

We compute that

$$
\begin{aligned}
\langle x(t)\rangle= & -\frac{1}{\delta}\left(\frac{x(t)-x(0)}{t}\right)-\frac{\omega}{\delta}\left(\frac{y(t)-y(0)}{t}\right)+\frac{\varepsilon}{\delta}+\frac{\sigma}{\delta t} \int_{0}^{t} x(s) y(s) d B(s) \\
& +\frac{\omega \alpha}{\delta}\langle y(t)\rangle-\frac{\omega \alpha \beta}{\delta}\left\langle y^{2}(t)\right\rangle \\
\leq & -\frac{1}{\delta}\left(\frac{x(t)-x(0)}{t}\right)-\frac{\omega}{\delta}\left(\frac{y(t)-y(0)}{t}\right)+\frac{\varepsilon}{\delta}+\frac{\sigma}{\delta t} \int_{0}^{t} x(s) y(s) d B(s) \\
& +\frac{\omega \alpha}{\delta}\langle y(t)\rangle-\frac{\omega \alpha \beta}{\delta}\left\langle\left. y(t)\right|^{2}\right. \\
\leq & -\frac{1}{\delta}\left(\frac{x(t)-x(0)}{t}\right)-\frac{\omega}{\delta}\left(\frac{y(t)-y(0)}{t}\right)+\frac{\varepsilon}{\delta}+\frac{\sigma}{\delta t} \int_{0}^{t} x(s) y(s) d B(s)+\frac{\omega \alpha}{4 \delta \beta} .
\end{aligned}
$$

Taking the limit superior on both sides of the above inequality yields

$$
\langle x(t)\rangle^{*} \leq \frac{\varepsilon}{\delta}+\frac{\omega \alpha}{4 \delta \beta} .
$$

Applying L'Hospital's rule to (24), we obtain $(x(t))^{*} \leq \frac{\varepsilon}{\delta}+\frac{\omega \alpha}{4 \delta \beta}$. Letting $r(t)=\alpha-x(t)$, with the condition of Theorem 3, we can derive that $r_{*} \geq \alpha-\left(\frac{\varepsilon}{\delta}+\frac{\omega \alpha}{4 \delta \beta}\right)>0$, applying this to (21) 
yields

$$
\begin{aligned}
d V_{2}(y(t)) & =\theta\left(1+V_{1}(y(t))\right)^{\theta-1}\left[-2 r(t) V_{1}(y)+2 \alpha \beta V_{1}^{0.5}(y)\right] d t \\
& \leq \theta\left(1+V_{1}(y(t))\right)^{\theta-1}\left[-2\left(r_{*}-\xi\right) V_{1}(y)+2 \alpha \beta V_{1}^{0.5}(y)\right] d t
\end{aligned}
$$

for sufficiently large $t \geq T$. Now, let $\eta>0$ be sufficiently small to satisfy $0<\eta / \theta<2\left(r_{*}-\xi\right)$. Define $V_{3}(y)=\exp (\eta t) V_{2}(y)$. Making use of Itô's formula gives

$$
\begin{aligned}
d V_{3}(y(t))= & \eta \exp (\eta t) V_{2}(y) d t+\exp (\eta t) d V_{2}(y) \\
\leq & \eta \exp (\eta t)\left(1+V_{1}(y)\right)^{\theta} d t+\exp (\eta t) \theta\left(1+V_{1}(y)\right)^{\theta-1}\left[-2\left(r_{*}-\xi\right) V_{1}(y)\right. \\
& \left.+2 \alpha \beta V_{1}^{0.5}(y)\right] d t \\
= & \theta \exp (\eta t)\left(1+V_{1} y\right)^{\theta-1}\left\{-\left[2\left(r_{*}-\xi\right)-\frac{\eta}{\theta}\right] V_{1} y+2 \alpha \beta V_{1}^{0.5}(y)+\frac{\eta}{\theta}\right\} d t \\
= & : \exp (\eta t) H(y) d t
\end{aligned}
$$

for $t \geq T$. Note that $H(y)$ is upper bounded in $R_{+}$, namely $C_{1}:=\sup _{y \in R_{+}} H(y)<+\infty$. Consequently,

$$
d V_{3}(y(t)) \leq C_{1} \exp (\eta t) d t
$$

for sufficiently large $t$. Integrating both sides of the above inequality yields

$$
\exp (\eta t)\left(1+y^{-2}(t)\right)^{\theta} \leq \exp (\eta T)\left(1+y^{-2}(T)\right)^{\theta}+\frac{C_{1}}{\eta}(\exp (\eta t)-\exp (\eta T)) .
$$

Then taking expectations on both sides of the above inequality gives

$$
E\left[\exp (\eta t)\left(1+y^{-2}(t)\right)^{\theta}\right] \leq \exp (\eta T)\left(1+y^{-2}(T)\right)^{\theta}+\frac{C_{1}}{\eta}(\exp (\eta t)-\exp (\eta T)) .
$$

We compute that

$$
\begin{aligned}
\limsup _{t \rightarrow \infty} E\left(1+y^{-2}(t)\right)^{\theta} & \leq \limsup _{t \rightarrow \infty}\left\{\frac{\exp (\eta T)\left(1+y^{-2}(T)\right)^{\theta}}{\exp (\eta t)}+\frac{C_{1}}{\eta}\left(1-\frac{\exp (\eta T)}{\exp (\eta t)}\right)\right\} \\
& \leq \frac{C_{1}}{\eta} .
\end{aligned}
$$

In other words, we have shown that

$$
\limsup _{t \rightarrow \infty} E\left[y^{-2 \theta}(t)\right] \leq \limsup _{t \rightarrow \infty} E\left(1+y^{-2}(t)\right)^{\theta} \leq \frac{C_{1}}{\eta}=: C .
$$

Thus, for any given $\xi>0$, let $N=\xi^{0.5 / \theta} / C^{0.5 / \theta}$, by Chebyshev's inequality,

$$
\mathcal{P}\{y(t)<N\}=\mathcal{P}\left\{y^{-\theta}>N^{-\theta}\right\} \leq \frac{E\left[y^{-2 \theta}(t)\right]}{N^{-2 \theta}}=N^{2 \theta} E\left[y^{-2 \theta}(t)\right] .
$$

That is to say, $\mathcal{P}^{*}\{y(t)<N\} \leq \xi$. Thus $\mathcal{P}_{*}\{y(t) \geq N\} \geq 1-\xi$. 
Theorem 4 If $\delta \alpha>\varepsilon$, then the tumor cells $y(t)$ will be strongly persistent in the mean almost surely.

Proof According to (23), we compute that

$$
\begin{aligned}
\langle x(t)\rangle= & -\frac{1}{\delta}\left(\frac{x(t)-x(0)}{t}\right)-\frac{\omega}{\delta}\left(\frac{y(t)-y(0)}{t}\right)+\frac{\varepsilon}{\delta}+\frac{\sigma}{\delta t} \int_{0}^{t} x(s) y(s) d B(s) \\
& +\frac{\omega \alpha}{\delta}\langle y(t)\rangle-\frac{\omega \alpha \beta}{\delta}\left\langle y^{2}(t)\right\rangle .
\end{aligned}
$$

Applying Itô's formula to the second equation of system (4) leads to

$$
d \ln y=(\alpha-\alpha \beta y-x) d t
$$

Integrating this from 0 to $t$ and dividing by $t$ on both sides, we have

$$
\frac{\ln y(t)-\ln y(0)}{t}=\alpha-\alpha \beta\langle y(t)\rangle-\langle x(t)\rangle .
$$

Substituting (25) to (26) yields

$$
\begin{aligned}
\frac{\ln y(t)-\ln y(0)}{t}= & \alpha-\alpha \beta\langle y(t)\rangle+\frac{1}{\delta}\left(\frac{x(t)-x(0)}{t}\right)+\frac{\omega}{\delta}\left(\frac{y(t)-y(0)}{t}\right)-\frac{\varepsilon}{\delta} \\
& -\frac{\sigma}{\delta t} \int_{0}^{t} x(s) y(s) d B(s)-\frac{\omega \alpha}{\delta}\langle y(t)\rangle+\frac{\omega \alpha \beta}{\delta}\left\langle y^{2}(t)\right\rangle \\
= & \alpha-\left(\alpha \beta+\frac{\omega \alpha}{\delta}\right)\langle y(t)\rangle+\frac{1}{\delta}\left(\frac{x(t)-x(0)}{t}\right)+\frac{\omega}{\delta}\left(\frac{y(t)-y(0)}{t}\right)-\frac{\varepsilon}{\delta} \\
& -\frac{\sigma}{\delta t} \int_{0}^{t} x(s) y(s) d B(s)+\frac{\omega \alpha \beta}{\delta}\left\langle y^{2}(t)\right\rangle . \\
\geq & \alpha-\left(\alpha \beta+\frac{\omega \alpha}{\delta}\right)\langle y(t)\rangle+\frac{1}{\delta}\left(\frac{x(t)-x(0)}{t}\right)+\frac{\omega}{\delta}\left(\frac{y(t)-y(0)}{t}\right)-\frac{\varepsilon}{\delta} \\
& -\frac{\sigma}{\delta t} \int_{0}^{t} x(s) y(s) d B(s) .
\end{aligned}
$$

We compute that

$$
\begin{aligned}
\langle y(t)\rangle \geq & \frac{\delta \alpha}{\alpha \beta \delta+\omega \alpha}+\frac{1}{\alpha \beta \delta+\omega \alpha}\left(\frac{x(t)-x(0)}{t}\right)+\frac{\omega}{\alpha \beta \delta+\omega \alpha}\left(\frac{y(t)-y(0)}{t}\right) \\
& -\frac{\varepsilon}{\alpha \beta \delta+\omega \alpha}-\frac{\sigma}{\alpha \beta \delta+\omega \alpha} \frac{1}{t} \int_{0}^{t} x(s) y(s) d B(s)-\frac{\delta}{\alpha \beta \delta+\omega \alpha} \frac{\ln y(t)-\ln y_{0}}{t} .
\end{aligned}
$$

Consider that both $x(t)$ and $y(t)$ are bounded, we have

$$
\lim _{t \rightarrow+\infty} \inf |y(t)| \geq \frac{\delta \alpha}{\alpha \beta \delta+\omega \alpha}-\frac{\varepsilon}{\alpha \beta \delta+\omega \alpha}=\frac{\delta \alpha-\varepsilon}{\alpha \beta \delta+\omega \alpha} .
$$

Consequently, we can derive that if $\delta \alpha>\varepsilon$, then $\langle y(t)\rangle_{*}>0$ a.s. 


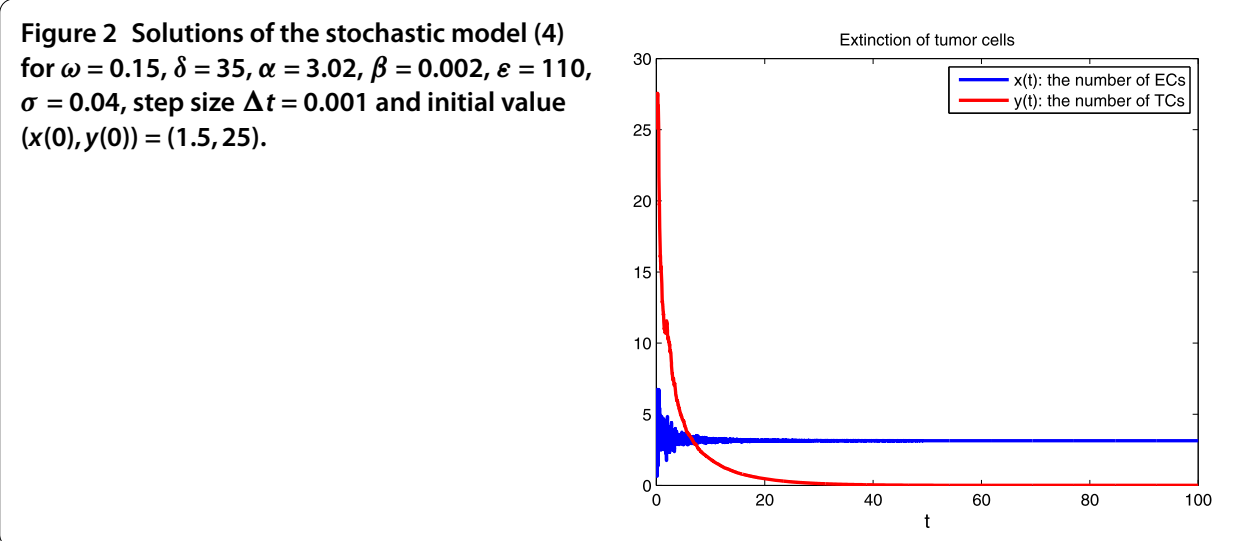

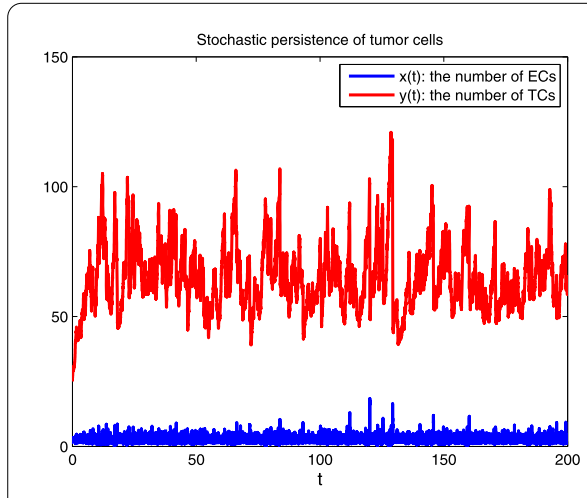

(a)

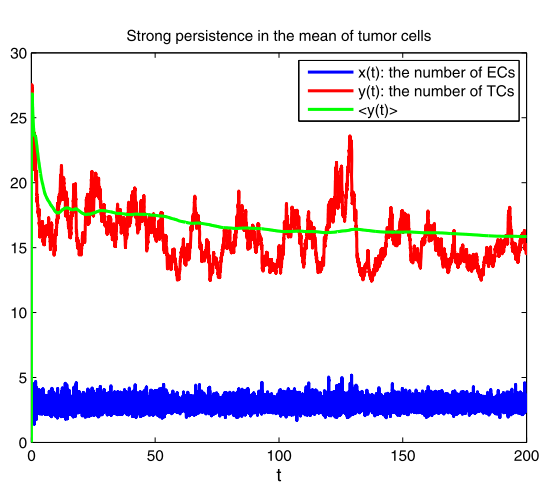

(b)

Figure 3 Solutions of the stochastic model (4) for $\omega=0.15, \delta=35, \alpha=3.02, \beta=0.002$, step size $\Delta t=0.006, \sigma=0.04$ and initial value $(x(0), y(0))=(1.5,25)$. (a) Is with $\varepsilon=83$; (b) is with $\varepsilon=100$.

\section{Numerical simulations}

In this section, we use the Euler-Maruyama numerical method mentioned by Higham [37] to support our results.

Figure 2 shows simulations of the results in Theorem 2. Based on the condition of Theorem 2, we choose $\varepsilon=110, \delta=35, \alpha=3.02$. Note that $\alpha \delta=105.7<\varepsilon=110$, and $\frac{\varepsilon}{\delta}=3.143$. Figure 2 demonstrates that tumor cells will tend to zero, and effector cells will tend to the constant 3.143 over time.

Figure 3 presents simulations of the results in Theorems 3 and 4. In Figure 3(a), we choose $\varepsilon=83, \delta=35, \alpha=3.02$. Then the conditions satisfy $\delta \alpha-\frac{\omega \alpha}{4 \beta}=86.825>\varepsilon=83$. Applying Theorem 3, we can see that $y(t)$ will be stochastically permanent. Figure 3(a) confirms the results. In Figure 3(b), we choose $\varepsilon=100, \delta=35, \alpha=3.02$. That is to say, $\alpha \delta=$ $105.7>\varepsilon=100$. By virtue of Theorem 4 , one can get that $y(t)$ will be strongly persistent in the mean and $\langle y(t)\rangle_{*} \geq \frac{\delta \alpha-\varepsilon}{\alpha \beta \delta+\omega \alpha}=15.7285$.

Figure 4 shows the effects of environmental noise on the mean time to the extinction of tumor-immune system competition system. Firstly, in Figure 4(a), we keep the parameter value $\varepsilon$ at 109 , which represents the normal rates of the flow of adult ECs into the tumor site, and simulate the path of $y(t)$ in both the stochastic model and its corresponding deterministic model. It is shown that $y(t)$ in the stochastic model and the deterministic 


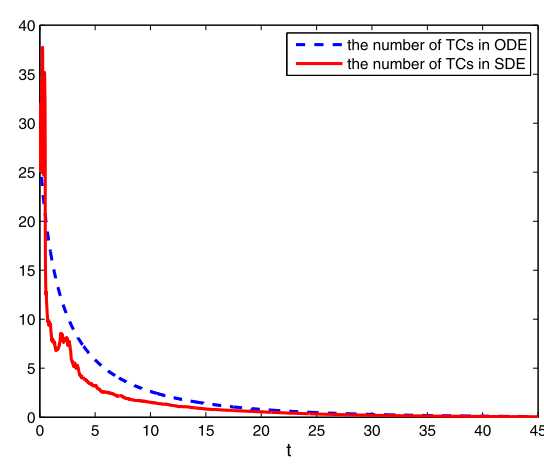

(a)

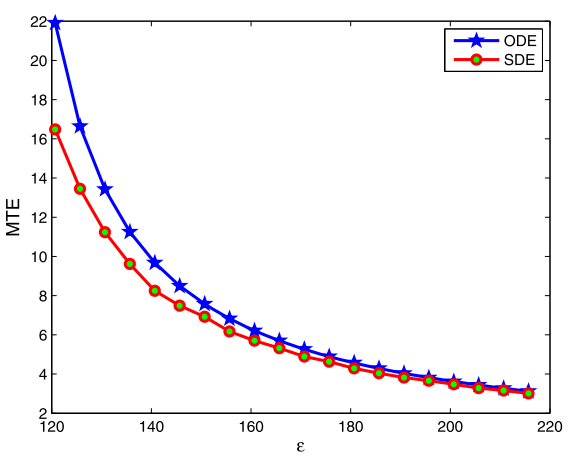

(b)

Figure 4 The effect of noise on the system for parameter values $\omega=0.15, \beta=0.001, \alpha=3.02, \delta=35$, $\sigma=0.3$, step size $\Delta t=0.01$ and initial value $(x(0), y(0))=(1.5,25)$. (a) is the path $y(t)$ for the stochastic model (4) and its corresponding deterministic model (3) with fixed parameter value $\varepsilon=109$; (b) is the mean time to extinction (MTE) as a function of $\varepsilon$.

model tends to zero since $\varepsilon=109>\alpha \delta=105.7$. However, the rate of tumor reduction in the stochastic model is faster than that in the deterministic model. These results can also be confirmed in Figure 4(b). Figure 4(b) simulates the mean time to extinction (MTE) as a function of $\varepsilon$, where the MTE is the average of 1,000 times of a sample path. It is obvious that the MTE in the stochastic model is less than that in the deterministic model when the values of $\varepsilon$ are the same, which shows that environmental noise can accelerate the extinction of tumor cells. In addition, it can be seen in Figure 4(b) that the MTE is reduced with the increase of $\varepsilon$, and it tends to almost the same value in the stochastic and deterministic models when $\varepsilon$ is relatively large. This result reveals that noise is favorable for the extinction of tumor cells under immune surveillance, and noise is ineffective when the ability of immune system is strong enough.

\section{Conclusion}

This paper is concerned with a stochastic tumor-immune system competition model. Firstly, the Kuznetsov-Taylor model and the Galash simplified model [9] describing the competition between the tumor and immune cells are introduced, Gaussian white noise is employed to mimic the environmental fluctuations. Then, with the methods of Itô's formula and Lyapunov function, sufficient conditions for extinction, stochastic persistence, and strong persistence in the mean of tumor cells are established by rigorous mathematical proofs. It is shown as follows:

(A) If $\alpha \delta<\varepsilon$, then the effector cells $x(t)$ go to $\frac{\varepsilon}{\delta}$, and the tumor cells $y(t)$ will go to extinction a.s.

(B) If $\alpha \delta-\frac{\omega \alpha}{4 \beta}>\varepsilon$, then the tumor cells $y(t)$ will be stochastically permanent a.s.

(C) If $\alpha \delta>\varepsilon$, then the tumor cells $y(t)$ will be strongly persistent in the mean a.s.

Our work reveals some important and interesting biological results. By comparing the results of Galach [9] with our work of Theorem 2, we can see that environmental noise can change the properties of tumor-immune population dynamics significantly. For example, under the same conditions, Figure 4(b) shows that the mean time to extinction of the stochastic model is less than the time in the deterministic model. Namely, environmental 
noise can accelerate the extinction of tumor cells under the surveillance of effector cells, which means that noise is favorable for the extinction of tumor in this condition.

Some interesting questions deserve further investigation. For example, in our model, we assume that fluctuations in the environment will mainly affect the immune coefficient. It is interesting to study what happens if noise affects other parameters of the system. Another question of interest is to consider the stability in distribution (e.g., [38, 39]). Moreover, one may propose some realistic but complex models. An example is to add the treatment into the system. The motivation is to predict the effect of treatment and design the optimal treatment schedule.

\section{Competing interests}

The authors declare that they have no competing interests.

\section{Authors' contributions}

$Y L$ proposed the model and drafted the main part of the manuscript. DL contributed to the revision of the manuscript and polished the language. All authors read and approved the final manuscript.

\section{Acknowledgements}

This work is supported by the National Natural Science Foundation of China (Grant Nos. 11402157 and 11571009), Shanxi Scholarship Council of China (Grant No. 2015-032), Technological Innovation Programs of Higher Education Institutions in Shanxi (Grant No. 2015121) and Applied Basic Research Programs of Shanxi Province (Grant No. 2016021013).

Received: 22 October 2016 Accepted: 7 February 2017 Published online: 21 February 2017

\section{References}

1. Cristini, V, Lowengrub, J: Multiscale Modeling of Cancer: An Integrated Experimental and Mathematical Modeling Approach. Cambridge University Press, Cambridge (2010)

2. Fory, U: Marchuk's model of immune system dynamics with application to tumor growth. J. Theor. Med. 4, 85-93 (2002)

3. Kirschner, DE, Panetta, JC: Modeling immunotherapy of the tumor-immune interaction. J. Math. Biol. 37, 235-252 (1998)

4. Mayer, H, Zaenker, KS, et al.: A basic mathematical model of the immune response. Chaos 5, 155-161 (1995)

5. Waniewski, J, Zhivkov, P: A simple mathematical model for tumor immune system interactions. In: The Eight National Conference on Application of Mathematics in Biology and Medicine, pp. 149-154 (2002)

6. Adam, JA, Bellomo, N: A Survey of Models for Tumor-Immune System Dynamics. Birkhauser, Boston (1997)

7. Eftimie, R, Bramson, JL, Earn, DJD: Interactions between the immune system and cancer: a brief review of non-spatial mathematical models. Bull. Math. Biol. 73, 2-32 (2011)

8. Kuznetsov, VA, Taylor, MA, et al.: Nonlinear dynamics of immunogenic tumors: parameter estimation and global bifurcation analysis. Bull. Math. Biol. 2, 295-321 (1994)

9. Galach, M: Dynamics of the tumor-immune system competition - the effect of time delay. Int. J. Appl. Math. Comput. Sci. 13, 395-406 (2003)

10. Forni, G, Giovarelli, M, et al.: Cytokine induced tumor immunogeneticity: from exogenous cytokines to gene therapy. J. Immunother. 14, 253-257 (1993)

11. Greller, LD, Tobin, FL, Poste, G: Tumor heterogenity and progression: conceptual foundation for modelin. Invasion Metastasis 16, 177-208 (1996)

12. Burger, R, Barton, NH: The Mathematical Theory of Selection, Recombination, and Mutation. Wiley, New York (2000)

13. Dieckmann, O, Heesterbeek, JAP: Mathematical Epidemiology of Infectious Diseases: Model Building, Analysis and Interpretation. Wiley, New York (2000)

14. May, R, Nowak, MA: Virus Dynamic: Mathematical Principles of Immunology and Virology. Oxford University Press, Oxford (2000)

15. Perelson, A, Weisbuch, G: Immunology for physicists. Rev. Mod. Phys. 69, 1219-1268 (1997)

16. Zhao, Y, Jiang, D, O'Regan, D: The extinction and persistence of the stochastic sis epidemic model with vaccination. Physica A 392, 4916-4927 (2013)

17. Wang, $Z, X u, Y, Y a n g, H$ : Levy noise induced stochastic resonance in an fhn model. Sci. China, Technol. Sci. 59, 371-375 (2016)

18. $X u, Y, L i, Y, Z h a n g, H, L i, X, K u r t h s, J:$ The switch in a genetic toggle system with Levy noise. Sci. Rep. 6, 31505 (2016)

19. $\mathrm{Xu}, \mathrm{Y}, \mathrm{Feng}, \mathrm{J}, \mathrm{Li}, \mathrm{J}, \mathrm{Zhang}, \mathrm{H}$ : Levy noise induced switch in the gene transcriptional regulatory system. Chaos 23 , $013110(2013)$

20. $\mathrm{Xu}, \mathrm{Y}, \mathrm{Wu}, \mathrm{J}, \mathrm{Du}, \mathrm{L}, \mathrm{Yang}, \mathrm{H}$ : Stochastic resonance in a genetic toggle model with harmonic excitation and Levy noise. Chaos Solitons Fractals 92, 91-100 (2016)

21. $\mathrm{Xu}, \mathrm{Y}$, et al.: Levy noise-induced stochastic resonance in a bistable system. Eur. Phys. J. B 86, 198 (2013)

22. $\mathrm{Xu}, \mathrm{Y}, \mathrm{Li}, \mathrm{Y}$, et al.: The phase transition in a bistable Duffing system driven by Levy noise. J. Stat. Phys. 158, 120-131 (2015)

23. $L i, Y, X u, Y$, Kurthsand, J, Yue, X: Levy noise induced transport in a rough triple-well potential. Phys. Rev. E 94, 042222 (2016) 
24. $\mathrm{Xu}, \mathrm{Y}, \mathrm{Gu}, \mathrm{R}, \mathrm{Zhang}, \mathrm{H}$ : Effects of random noise in a dynamical model of love. Chaos Solitons Fractals 44, 490-497 (2011)

25. $\mathrm{Xu}, \mathrm{Y}, \mathrm{Wang}, \mathrm{H}, \mathrm{Liu}, \mathrm{D}, \mathrm{Huang}, \mathrm{H}$ : Sliding mode control of a class of fractional chaotic systems in the presence of parameter perturbations. J. Vib. Control 21, 435-448 (2015)

26. Zhong, W, Shao, Y, He, Z: Pure multiplicative stochastic resonance of a theoretical antitumor model with seasonal modulability. Phys. Rev. E 73, 95 (2006)

27. Albano, G, Giorno, V: A stochastic model in tumor growth. J. Theor. Biol. 242, 329-336 (2006)

28. Lenbury, Y, Triampo, W, Tang, IM, et al.: A stochastic model of cancer growth with immune response. J. Korean Phys. Soc. 49, 1652-1666 (2006)

29. Ferrante, L, Bompadre, S, et al.: Parameter estimation in a Gompertzian stochastic model for tumor growth. Biometrics 56, 1076-1081 (2006)

30. Xu, Y, Feng, J, Li, J, Zhang, H: Stochastic bifurcation for a tumor-immune system with symmetric Levy noise. Physica A 392, 4739-4748 (2013)

31. Fiasconaro, A, Spagnolo, B: Co-occurrence of resonant activation and noise-enhanced stability in a model of cancer growth in the presence of immune response. Phys. Rev. E 74, 159 (2006)

32. Liu, M, Fan, M: Permanence of stochastic Lotka-Volterra systems. J. Nonlinear Sci. (2016). doi:10.1007/s00332-016-9337-2

33. Liu, M, Wang, K: Persistence and extinction in stochastic non-autonomous logistic systems. J. Math. Anal. Appl. 375, 443-457 (2011)

34. Mao, X, Marion, G: Environmental Brownian noise suppresses explosions in populations dynamics. Stoch. Process. Appl. 97, 95-110 (2002)

35. Evans, LC: An Introduction to Stochastic Differential Equations. Am. Math. Soc., New York (2013)

36. Mao, X: Stochastic Differential Equations and Applications. Horwood, Chichester (1997)

37. Higham, DJ: An algorithmic introduction to numerical simulation of stochastic differential equations. SIAM Rev. 43, 525-546 (2001)

38. Liu, M, Bai, C: Dynamics of a stochastic one-prey two-predator model with Levy jumps. Appl. Math. Comput. 284 308-321 (2016)

39. Liu, M, Bai, C: Optimal harvesting of a stochastic mutualism model with Levy jumps. Appl. Math. Comput. 276 301-309 (2016)

\section{Submit your manuscript to a SpringerOpen ${ }^{\ominus}$ journal and benefit from:}

- Convenient online submission

- Rigorous peer review

- Immediate publication on acceptance

- Open access: articles freely available online

- High visibility within the field

- Retaining the copyright to your article 